\title{
Unbefriedigende Behandlung der Depression - nur Sache des Hausarztes?
}

\section{Pierre Loeb*}

* Im Namen von «Hausärzte Schweiz» und der Schweizerischen Akademie für Psychosomatische und Psychosoziale Medizin SAPPM. Der Autor ist Präsident der SAPPM und in der psychosozialen Fortbildung von Grundversorgern aktiv.
Korrespondenz: Dr. med. Pierre Loeb Winkelriedplatz 4 CH-4008 Basel

loeb@hin.ch
Hausärzte behandeln ein Drittel bis die Hälfte aller depressiven Erwachsenen, bzw. zwei Drittel der depressiven alten Patienten. Die Schwere der Symptome der Depression sind in Hausarzt-Praxen und bei FachPsychiatern vergleichbar [2]. Neue und sehr grosse Studien [3] belegen, dass «im Allgemeinen ein motivierter Grundversorger in einem städtischen (amerikanischen) Umfeld (wo die Depressionsrate bei 20\% liegt) 10 von 20 Fällen korrekt erkennt und dabei zehn Positive verpasst (mittlere Sensitivität). Er wird aber 65 von 80 nichtdepressiven Personen zu Recht beruhigen (hohe Spezifität) [4] und 15 Personen fälschlich die Diagnose depressiv geben.»

Tyrer [5] diskutiert in einer im August 2009 erschienen Publikation im Lancet die Studie von Mitchell [3] und fragt sich, was in der letzten Generation bei den Hausärzten betreffend Behandlung der Depression geschehen ist. Dabei evaluiert er die Kompetenz des Hausarztes und den Aussagewert der Screeningfragen und gelangt zum Schluss, dass die Einschätzung des Gesamtbildes durch den Hausarzt den Screeningfragen sogar überlegen sein könnte. Vor allem wiederholtes Nachfragen bringe mehr als einmaliges Screening. Depressive Patienten verschleiern oder spielen ihre Symptome wegen der Stigmatisierung der Diagnose Depression gern herunter und noch immer herrscht eine grosse Angst vor, von Antidepressiva «süchtig» zu werden oder nicht mehr sich selbst zu sein [6]. Viele Patienten erzählen nicht spontan von ihrer niedergedrückten Stimmung, sondern bieten stattdessen multiple körperliche Symptome an, die sie von ihrem Hausarzt behandelt wissen wollen. Kielholz [7] sprach schon 1981 von der larvierten〉 Depression. Die allgemein typische Depressionssymptomatik entspricht der weiblichen Form. Männer neigen eher dazu, ihre affektive Störung zu kaschieren. Sie ziehen sich zurück, ertränken ihren Kummer im Alkohol und verlieren die Kontrolle mit abrupten Wutanfällen oder Gewaltausbrüchen. Gerade diese Eigenschaften sind so peinlich, dass sie allzu oft weder von den Patienten noch von ihren Partnerinnen früh genug geäussert werden. Burnout, Mobbing und vor allem Angststörungen sind oft Diagnosen, die der Hausarzt als Umschreibung einer Depression bewusst verwendet, um die Patienten nicht zu stigmatisieren, sondern sie für eine therapeutische Beziehung zu gewinnen, bzw. weil diese primär wünschen, dass die vorherrschenden
Symptome angegangen werden. So kann es nicht erstaunen, dass ein Grossteil der Depressionen nicht von Anfang an als solche deklariert wird. Handelt es sich um eine rezidivierende Episode einer Depression oder erkennt der Patient seine Krankheit und wünscht

\section{Zum «Positionspapier zur Verdoppelung des Selbstbehaltes für psychisch Kranke» der FMPP}

Hausärzte und Psychiater sind seit zwei Jahren im Aktionsbündnis psychische Gesundheit Schweiz sowie im Bündnis gegen Depression um nachhaltige Lösungen zur Optimierung von Erkennung, Behandlung und Betreuung von Menschen mit Depression und Suizidalität bemüht. Aus dieser Sicht einer breit abgestützten Basisversorgung durch verschiedene Fachorganisationen der Ärzteschaft (Hausärzte und Psychiater), Psychologen und den kantonalen Versorgungsstrukturen erstaunt das «Positionspapier zur Verdoppelung des Selbstbehaltes für psychisch Kranke» der Psychiatervereinigung FMPP in der SÄZ Heft 49/2009 [1]. Zwar gestehen die Autoren den «Hausärzten (...) heute gute Kenntnisse in Psychiatrie» zu, geben aber gleichzeitig zu bedenken "Grundversorger sind wichtig, jedoch keinesfalls ausreichend.» Gleichzeitig vermitteln sie beängstigende Resultate: «Trotz der Häufigkeit psychischer Erkrankungen werden diese oft nicht als solche diagnostiziert und entsprechend behandelt» (...) «1300 Menschen sterben jährlich in der Schweiz an Suizid, ca. 15000 Menschen in der Schweiz begehen einen Suizidversuch» und «über die Hälfte der sich suizidierenden Menschen sucht noch im Monat vor dem Ereignis ihren Hausarzt auf.» Sind die Hausärzte dafür verantwortlich?

Zwei grosse Studien und Reviews mit Fragestellungen zu Erkennung und Outcome der Depression sind 2009 erschienen: «Clinical diagnosis of depression in primary care: a meta-analysis» von Alex Mitchell et al. im Lancet [3] und «Screening for depression in adult patients in primary care settings: a systematic evidence review» von O,Connor et al. in Annals of Internal Medicine [9]. 
den raschen Zugang zu (s)einem Psychiater, muss ihm dies garantiert sein, damit keine unnötige Zeit verlorengeht.

Betrachtet man die Konsultationen im Längsschnitt, wird dem Hausarzt die Entwicklung einer Depression je länger, desto weniger entgehen [8], und auch die Akzeptanz beim Patienten nimmt zu, wurde er a) zuerst somatisch korrekt abgeklärt und b) fehlt die Besserung seines Gemütszustands trotz anderweitiger Behandlung. Dennoch muss hier selbstkritisch gesagt werden, dass es Hausärzte gibt, die zu lange die depressive Ursache ausser Acht lassen und damit sowohl den Beginn der medikamentösen Behandlung als auch den Einbezug der psychosozialen Dimension hinauszögern.

Brandaktuell, nämlich vom Dezember 2009, ist eine methodisch perfekt gemachte Review (samt der für die Thematik relevanten Primärliteratur) von engere Überwachung der Behandlung (z.B. durch Telefonanrufe), Prüfung von Verträglichkeit und eine psychosoziale Grundbetreuung durchzuführen. Solche «disease management» [11] und «collaborative care» sind wirksam - führen aber zu höheren Gesundheitskosten [12]. Die Entschädigung dieser Art von Mitarbeit unserer MPAs ist bei uns im Tarif (noch) nicht vorgesehen, obwohl die Erfolgsrate hiermit wohl am effizientesten zu beeinflussen wäre.

Die adäquate Behandlung des Depressionszustands ist wahrscheinlich von grösserer Bedeutung als die ICD-10- oder DSM-IV-konforme Formulierung der Diagnose. Trotz der Wirksamkeit und dem verbesserten Nebenwirkungsspektrum der Antidepressiva werden diese nach wie vor unzuverlässig eingenommen. Die Angst vor Nebenwirkungen und die Angst davor, von den Medikamenten süchtig zu werden, sind die Gründe dafür, dass 40 bis 67 Prozent der Pa-

\section{Neue Fortbildungs-, Versorgungs- und Zusammenarbeitsmodelle, wie sie im Bündnis gegen Depression vorgesehen sind, sollen die Betreuung Depressiver und die Suizidprävention verbessern}

$\mathrm{O}^{\prime}$ Connor und Mitarbeitern in den Annals of Intern Medicine [9]. Hier wird die Bedeutung des Screenings der Depression, das 2002 von der U.S. Preventive Services Task Force (USPSTF) empfohlen und 2005 von Cochrane [10] relativiert wurde, neu überprüft.

Es mag erstaunen, dass obwohl das Screening zu einer besseren Erkennungsrate von Depressionen führt, es jedoch keine Besserung des Krankheitsverlaufs nach sich zieht. Eine Erklärung - so O'Connor liegt darin, dass vom Hausarzt nicht erkannte Depressionen weniger schwer verlaufen als diejenigen, die auch ohne Screening entdeckt werden. Viele dieser Störungen heilen spontan und ohne medikamentöse Therapie aus. Weiter folgert $\mathrm{O}^{\prime}$ Connor, dass hausärztliche Depressions-Screeningprogramme nur dann Erfolg haben, wenn eine entsprechende Praxisinfrastruktur zur Verfügung steht, die neben der Diagnosensicherung auch eine individuelle Patientenbetreuung, Therapiemöglichkeit und Nachsorge sicherstellt. Gensichen [11] konnte für Deutschland nachweisen, dass Case Management durch Medizinische Praxisassistent(inn)en (MPA) in Grundversorgerpraxen (Small Primary Care Practices) die Einhaltung der Therapie (increased treatment adherence) und den Verlauf der Depression günstig beeinflussen konnte.

In naher Zukunft könnten neue Versorgungsmodelle wie Gruppenpraxen, die über eine Infrastruktur mit Gesundheitsfachleuten sowie Fachpsychotherapeuten oder Fachärzte verfügen, eine umfassende Therapie sicherstellen. Aber schon jetzt könnten mit Hilfe des bestehenden Praxisteams bessere Resultate erreicht werden, wenn dieses befähigt wird, eine tienten ihre medikamentöse Behandlung innert drei Monaten [10] sistieren, obwohl die Medikamente 6 bis 12 Monate nach Erreichen der Besserung eingenommen werden sollten. Hierfür sind Aufklärung, Begleitung, Anpassung der Dosierung oder evtl. Kombination sehr wichtig.

Die zweite Schiene der Behandlung liegt in der begleitenden Gesprächstherapie - die auch für eine konsequente Medikamenteneinnahme sorgen könnte. Hier bestehen die wichtigsten Hindernisse im Zeitmanagement in den Arztpraxen, wo die notwendigen «Randstunden» für die stützenden ärztlichen Gespräche oft fehlen, finanziell schlecht honoriert werden und für manchen Hausarzt sehr belastend sind. Speziell für solche Fälle bietet die SAPPM eine Weiterbildung an, in der die Kompetenz u.a. im Umgang mit «schwierigen» Patienten erworben werden kann. Neben Theorie und speziellen Fertigkeiten werden Supervision in einem spezialisierten Qualitätszirkel oder in einer Balintgruppe angeboten.

Die stützenden bzw. psychosomatischen Therapien liegen jedoch nicht allen Hausärzten, so dass sich dann die Zuweisung an einen Psychotherapeuten/ Psychiater aufdrängt. Für den sinnvollen und erwünschten niederschwelligen Zugang zum Psychiater/Psychotherapeuten zeigen sich regional enorme Unterschiede. Gewisse Landesteile haben gute Vernetzungen mit den regionalen Institutionen oder verfügen sogar über mobile Equipen, so dass eine schnelle Übernahme durch einen Psychiater vor allem in Notfällen durchaus gewährleistet ist. In vielen Regionen ist die psychiatrische Versorgung je- 
doch ungenügend und ein eigener psychiatrischer Notfalldienst fehlt. Praktisch überall hapert es leider bei der Zuweisung zu einer psychotherapeutischen Behandlung innerhalb einer akzeptablen Frist. Barrieren bestehen nicht nur in den kurzen und seltenen Telefonpausen und langfristig besetzten Terminen, sondern auch in Sprachproblemen und teils fehlender gegenseitiger Akzeptanz - gerade bei somatisierenden Patienten.

Es wird weltweit damit gerechnet, dass sich die Depression zur zweithäufigsten Krankheit überhaupt entwickelt. Um beim Aufbau eines fachübergreifendes Netzwerkes mit Hausärzten, Psychiatern und weiteren regionalen Institutionen mit dem Ziel einer besseren Versorgung mitzuhelfen, hat das Bundesamt für Gesundheit das international erfolgreiche Modell des Bündnis gegen Depression für die Schweiz übernommen. In den Kantonen Zug, Bern, Thurgau und Luzern sind solche Netzwerke zur Verbesserung der Versorgungs- und Lebenssituation von depressiv erkrankten Menschen und ihren Angehörigen sowie zur Suizidprävention bereits erfolgreich eingeführt. Weitere Kantone werden hinzukommen. Um dieser Herausforderung besser gerecht zu werden, arbeiten BAG, KHM und SAPPM zusammen an einem Kompetenzprofil für die hausärztliche Praxis. Ziel ist die Optimierung von Diagnostik, Therapie und Prävention sowie der Versuch, die Depression zu enttabuisieren und im Bündnis erfolgreicher zu behandeln. Die Wiedererlangung der Gesundheit und der damit verknüpften Arbeitsfähigkeit braucht eine gute Zusammenarbeit zwischen Hausärzten und Psychiatern wir sind bereit dazu.

\section{Zusammenfassung}

- Hausärzte behandeln ein Drittel bis die Hälfte aller depressiven Erwachsenen bzw. zwei Drittel der alten depressiven Patienten.

- Neuere Studien interpretieren die Erfolgsrate des Screenings in der Praxis differenzierter und zeigen, dass wiederholtes Nachfragen mehr bringt als einmaliges Screening.

- Erfolg zeigen Praxisteams (bestehend aus MPA bis Careteam), die zu verbesserter Behandlungstreue (increased treatment adherence) führen.

- Erreicht wird dies durch Einhaltung und engere Überwachung der Behandlung sowie psychosozialer Betreuung.

- «Disease management» und «collaborative care» sind wirksam - führen aber zu höheren Gesundheitskosten.

- Der Zugang zu Psychiatern (im Notfall) wie auch zur Pharmako- und Psychotherapie bei komplexen Fällen muss verbessert werden.
- Neue Fortbildungs-, Versorgungs- und Zusammenarbeitsmodelle, wie sie im Bündnis gegen Depression vorgesehen sind, sollen die Betreuung Depressiver und die Suizidprävention verbessern.

Ich danke Ueli Grüninger vom Kollegium für Hausarztmedizin KHM und Fränzi Zogg und Margot Enz vom Vorstand Hausärzte Schweiz für die wertvollen Impulse und das kritische Gegenlesen.

\section{Literatur}

Die hier aufgeführten Untersuchungen stammen nicht aus der Schweiz und lassen sich somit nur bedingt auf unsere Verhältnisse übertragen.

1 Ebner G und Kurt H. Positionspapier zur Verdoppelung des Selbstbehaltes für psychisch Kranke. Schweiz Ärztezeitung. 2009;90(49):1920-22.

2 Gaynes BN, Rush AJ, Trivedi M, Wisniewski SR, Balasubramani GK, Spencer DC et al. A direct comparison of presenting characteristics of depressed outpatients from primary vs. specialty care settings: preliminary findings from the STAR*D clinical trial. Gen Hosp Psychiatry. 2005;27:87-96. [PMID: 15763119].

3 Mitchell AJ, Vaze A, Rao S. Clinical diagnosis of depression in primary care: a meta-analysis. Lancet 2009; published online July 28. DOI:10.1016/ S0140-6736(09)60879-5.

4 Monica Cepoiu et al. Recognition of Depression by Non-psychiatric Physicians - A Systematic Literature Review and Meta-analysis (JGIM 2007) <JGIM Cepoiu_11606_2007_Article_428.pdf>

5 Tyrer P. Are general practitioners really unable to diagnose depression? Lancet. Vol. 374. August 22. 200

6 Khan N, Bower P, Rogers A. Guided self-help in primary care mental health: meta-synthesis of qualitative studies of patient experience. Br J Psychiatry. 2007;191:206-11.

7 Kielholz P, Pöldinger W, Adams C. Die larvierte Depression. Köln: Dt. Ärzte-Verlag; 1981.

8 Cooper B. A study of one hundred chronic psychiatric patients identified in general practice. $\mathrm{Br} J$ Psychiatry. 1965; 11:595-605.

9 O'Connor et al. Screening for depression in adult patients in primary care settings: a systematic evidence review. Ann Intern Med. 2009; Dec 1.151(11):793-803.

10 Gilbody et al. Cochrane Database Syst Rev. 2005; Oct 19;(4):CD002792.

11 Gensichen J et al. Case Management for Depression by Health Care Assistants in Small Primary Care Practices. A Cluster Randomized Trial. Annals of Internal Medicine. Sept 2009.

12 Suresh AJ. ebmh.bmj.com 